\title{
Die Schweiz und der internationale Kampf zur Beendigung der Tabakepidemie
}

\author{
Seit Jahrzehnten besteht eine tödliche Epidemie, gegen die bisher nur zögerlich \\ vorgegangen worden ist, obwohl sie in der Schweiz jährlich Tausende und weltweit \\ Millionen Menschenleben fordert. 178 Staaten haben sich durch Ratifikation einer \\ Rahmenkonvention verpflichtet, an der gemeinsamen Besiegung mitzuarbeiten. \\ Doch die Schweiz steht bisher abseits.
}

\section{Theodor Abelin}

1971-2000 Professor und Direktor des Instituts für Sozialund Präventivmedizin der Universität Bern, 1973-1992 Präsident der 1973 gegründeten Arbeitsgemeinschaft Tabakprävention Schweiz, 2001-2004 Präsident der Weltföderation der Public-Health-Gesellschaften
Korrespondenz: Prof. em. Dr. med. Theodor Abelin Seelandstrasse 23 CH-3095 Spiegel b. Bern Tel. 0319710159

th.abelin[at]bluewin.ch

\section{Nikotinsucht und Anfixen als Schlüssel}

Der Erreger der tödlichen Epidemie ist der Tabakrauch, der jahrzehntelang inhaliert wird, weil ihre Konsumenten süchtig sind - süchtig geworden als Kinder und Jugendliche und angefixt durch eine raffinierte Kombination von Werbung, Promotion und Sponsoring, die darauf ausgerichtet ist, sie glauben zu machen, Rauchen sei unter Erwachsenen etwas «Normales» und Trendiges.

Das Suchtpotential von Nikotin ist vergleichbar mit demjenigen von Kokain. Bei vielen Jugendlichen löst das Rauchen einiger weniger Zigaretten im Hirn ein langfristiges Verlangen nach Nikotin aus. Und Nikotinabhängigkeit erleichtert die Entwicklung von Kokainabhängigkeit [1]. Bei der Förderung des Erstkonsums geht es also um Anfixen, und solange angefixt wird, setzt sich die Epidemie fort. Die interessierten Kreise haben bisher vom Aspekt der Suchtwirkung des Nikotins abgelenkt und mit dem Recht des Erwachsenen zu rauchen argumentiert. Doch um diese Frage geht es hier nicht. Erwachsene Raucher dürfen ruhig weiterhin rauchen, solange es ihre Mitmenschen nicht stört oder gefährdet und den Jungen nicht als Vorbild dient. Sie müssen Tabakprodukte kaufen können, und im Interesse ihrer Gesundheit muss ihnen Hilfe beim Aufhören angeboten werden.

\section{Das Tabakproduktegesetz als Basis der Epidemiebekämpfung}

In der Epidemiebekämpfung sind zur Eliminierung der Krankheitsursache gelegentlich repressive Massnahmen angezeigt. Das Tabakproduktegesetz (TPG) erlaubt solche, ebenso wie das Gift- oder das Heilmittelgesetz, die ebenfalls den Handel mit Produkten mit Schädlichkeitspotential regeln, und im Gegensatz zum bisher als Rahmen benützten Lebensmittelgesetz. Damit ist endlich die gesetzgeberische Voraussetzung für eine erfolgreiche «Denormalisierung» des Rauchens und Beendigung der Tabakepidemie gegeben.

\section{Considérations en vue de la loi sur les produits du tabac}

Dans le contexte de la consultation en cours sur la nouvelle loi relative aux produits du tabac, l'auteur propose de miser sur une stratégie visant à enrayer l'épidémie de tabagisme en empêchant toute incitation des jeunes à fumer. Si cette stratégie ne s'adresse pas aux fumeurs, elle a pour objectif d'éviter que les jeunes considèrent l'acte de fumer comme un acte «normal» dans le monde des adultes. Cela suppose une interdiction stricte de toute forme de publicité, de promotion et de sponsoring en remplaçant, entre autre, le financement de la culture et du sport par l'industrie du tabac par une hausse des moyens issus du fonds de prévention du tabagisme. Les cigarettes électroniques devront être mises sur le même plan que les cigarettes afin d'éviter tout contournement de cette interdiction. Jusqu'ici, la législation suisse a permis aux multinationales du tabac d'éviter d'avoir à mettre en place les mesures préventives de la Convention-cadre de l'OMS pour la lutte antitabac. Or, le nouveau texte de loi est formulé de telle manière qu'il permettra également à cette pratique de perdurer au détriment de la santé dans les pays défavorisés. Pour des raisons de solidarité mais également pour préserver la réputation de la Suisse à l'échelle internationale, l'auteur estime donc qu'une modification du texte de loi s'impose. Enfin, l'auteur met en lumière les avantages d'une initiative populaire par rapport à un référendum dans le cas où la nouvelle loi ne satisferait pas aux exigences permettant d'enrayer l'épidémie de tabagisme. 


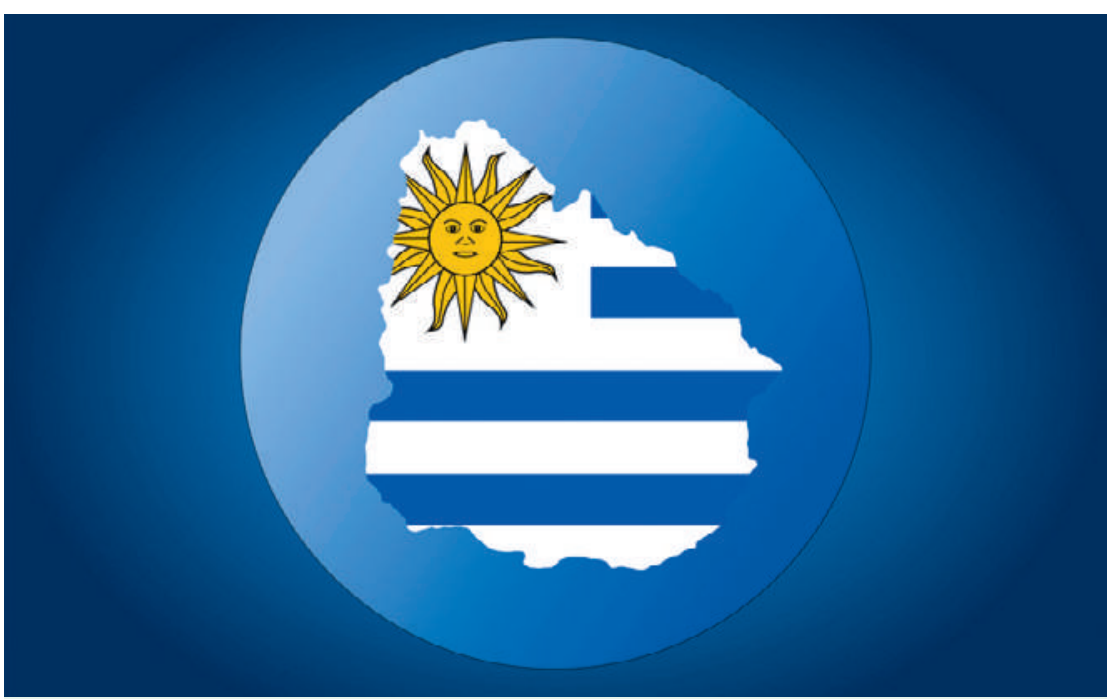

Im Fokus der Tabakindustrie: Uruguay, denn es wehrt sich gegen den Import besonders schädlicher Zigaretten. Aufgrund der jetzigen Gesetzeslage kann Philip Morris von der Schweiz aus dagegen klagen.

\section{Welche Massnahmen sind erforderlich?}

Zur Verhinderung des Anfixens Jugendlicher ist ein generelles und bedingungsloses Verbot von Werbung, Promotion und Sponsoring unentbehrlich - allenfalls mit der Ausnahme der Information des Tabakhandels. In einer Studie der Eidgenössischen Kommission für Tabakprävention (EKTP) [2] mit über 60 zitierten Literaturreferenzen haben wir gefunden, dass zur

\section{Ein generelles und bedingungsloses Verbot von Werbung, Promotion und Sponsoring ist unentbehrlich.}

Vermeidung des Erstkonsums nur ein lückenloses Verbot wirksam ist, da partielle Verbote stets Umgehungsmöglichkeiten bieten. Andererseits bleiben die meisten erwachsenen Raucher/innen zeitlebens ihrer Marke treu, so dass die Werbung ihre Markenwahl kaum mehr beeinflusst. Von Tabakwerbung zu sprechen, die sich an Erwachsene richtet, ist deshalb irreführend und gehört nicht in den Gesetzestext.

\section{Andere Lösung für das Sponsoring von Anlässen}

Gemäss dem Entwurf soll das Sponsoring für Tabakprodukte und -marken für Schweiz-interne Anlässe zugelassen werden. Begründet wird dies durch die andernfalls drohende Gefährdung wichtiger sportlicher und kultureller Veranstaltungen. Das könnte jedoch durch eine mit dem Tabakproduktegesetz verbundene kleine Revision der Verordnung über den Tabakpräventionsfonds (TPFV) vermieden werden, indem der Tabakpräventionsrappen von heute 1,3 Rappen pro verkaufte Zigarettenpackung entsprechend dem für das Sponsoring erforderlichen Betrag erhöht wird. (Weitere 1,3 Rappen dienen der Unter- stützung des Tabakanbaus.) In der Romandie führen die «Centres d'information pour la prévention du tabagisme» (CIPRET) schon seit Jahren ein Sponsoring in diesen Bereichen durch, und dieses könnte mit den zusätzlichen Mitteln ausgebaut und auf die gesamte Schweiz erweitert werden.

\section{E-Zigaretten bedrohen die Bekämpfung der Tabakepidemie}

E-Zigaretten ermöglichen eine Nikotinaufnahme durch Dampf ohne Inhalation der gesundheitsschädlichen Rauchbestandteile des Tabaks. In den letzten Jahren haben sich die grossen Tabakkonzerne in den E-Zigarettenmarkt eingekauft, und seither wurde der Aufwand zur Bekanntmachung dieser Produkte vervielfacht. Während die Frage der Schädlichkeit der Dampfbestandteile noch nicht abschliessend geklärt ist, ist klar, dass E-Zigaretten missbraucht werden können, um Werbeverbote für Tabakwaren zu umgehen und der angestrebten Denormalisierung des Rauchens entgegenzuwirken. Eine führende Bierbrauerei demonstrierte dies vor einigen Jahren, indem sie unter Umgehung des damaligen Verbots der TV-Werbung für alkoholische Getränke TV-Spots mit typischer Bierwerbung und ihrem Marken-Logo ausstrahlte und am Schluss kurz einblendete, dass es sich um alkoholfreies Bier handle. Die vorgeschlagene konsequente Einbeziehung der E-Zigaretten unter das Tabakproduktegesetz ist daher unentbehrlich - umso mehr als auch E-Zigaretten ein Anfixen mit Nikotin ermöglichen. Deshalb wäre auch vorzuziehen, für die Nikotinaufnahme anstelle der E-Zigaretten Behälter in der Form von Medikamenten- oder Giftfläschchen vorzuschreiben. Dafür spricht auch, dass das Nikotin bei unsorgfältigem Nachfüllen der Verdampfungsgeräte auslaufen und als starkes Gift wirken kann.

\section{Markierung von Zigaretten im Kampf gegen den internationalen Schmuggel}

In wirtschaftlich wenig entwickelten Ländern erfolgt ein beträchtlicher Teil des Anfixens mit gesetzeswidrig gehandelten Zigaretten, und damit verbunden ist eine Bindung der Bevölkerung an die angebotenen Marken. Wie mehrfach gezeigt wurde, sind die internationalen Tabakkonzerne dabei direkt beteiligt, und der Schweiz kommt als Drehscheibe des Transports und der Finanzflüsse eine bedeutende Rolle zu [3, 4]. Diesen Handel zu unterbinden ist unentbehrlich, um die Verbreitung der Tabakepidemie in ärmere Länder zu verhindern. Und das zentrale Instrument ist das Track-and-Tracing-System im Rahmen der von 178 Staaten ratifizierten Rahmenkonvention zur Tabakkontrolle, das erlauben soll, alle gesetzeskonform gehandelten Zigaretten einheitlich zu bezeichnen, auf ihrem Weg durch die Welt zu verfolgen und festzustellen, wo sie allenfalls vom legalen Transportweg abgewichen sind. Dazu müssen die Staaten die Daten einheitlich erheben und einer zentralen Datenbank zuführen. Die vom Bundesrat vorgeschlagene Verwendung eines eigenen Codes durch 
die Schweiz würde die internationale Zusammenarbeit in folgenschwerer Weise zuungunsten der Entwicklungsländer unterwandern, wie der Autor letzthin auch am Sitz der WHO in Genf hören konnte. Denn nur wenn die Schweiz hier mitarbeitet, kann das internationale Ziel erreicht werden, die Entwick-

\section{«Wollen wir wirklich eine Schweiz, die gezielt ermöglicht, die Prävention chronischer Krankheiten in anderen Ländern zu behindern?»}

\section{Literatur}

1 Levine A et al. Molecular Mechanism for a Gateway Drug: Epigenetic Changes Initiated by Nicotine Prime Gene Expression by Cocaine. Sci Transl Med. 2011;3.107ra109.

2 Neue Erkenntnisse zu Marketing und Werbung bei Tabakerzeugnissen. Eine Übersicht zu gesetzlichen Massnahmen und ihrer Wirksamkeit. Th. Abelin, Valérie Borioli Sandoz, Claude Jeanrenaud. Eidg. Departement des Innern EDI. Eidg. Kommission für Tabakprävention. Bern; 2011.

3 Joossens L, Raw M. Progress in combating cigarette smuggling: controlling the supply chain. Tob Control. 2008;17:399-404.

4 Joossens L, Raw M. From cigarette smuggling to illicit tobacco trade. Tob Control. 2012;21:230-4.

5 Häne S. In der Schmuggelzone. Tages-Anzeiger / Der Bund, 31.5.2014.

6 Alliance Sud, 20.7.2013 (www.alliancesud.ch/de/ep/ handel/philip-morris gegen-uruguay-1-0) abgerufen 15.6.2014) lungsländer vor der Ausbreitung der tödlichen und für ihr Gesundheitswesen unbezahlbaren Tabakepidemie zu schützen. So geht es hier um eine zentrale Frage der Reputation der Schweiz in der internationalen Staatengemeinschaft und nicht nur um eine Teilfrage der Problematik Schweiz/EU [5]. Eine Änderung in Art. 35 des Entwurfs könnte diese Zusammenarbeit ermöglichen, etwa mit einer Formulierung wie: «Die Schweiz nimmt am internationalen Datenaustausch teil, wenn dieser gesundheitlichen Zwecken, insbesondere der Bekämpfung des illegalen Handels dient.»

\section{Export von speziell schädlichen Zigaretten in Länder, die diese nicht importieren wollen}

In Übereinstimmung mit der internationalen Praxis enthält der Entwurf für die Schweiz ein Verbot der Abgabe von Zigaretten, deren Rauch den Gehalt von $10 \mathrm{mg}$ Teer, 1,0 mg Nikotin und $10 \mathrm{mg}$ Kohlenmonoxid übersteigt, doch verzichtet er auf ein generelles Produktions- und Exportverbot für solche Zigaretten, um, wie es weltweit üblich ist, auch die Bevölkerung anderer Länder zu schützen. Dies sollte korrigiert werden.

Ein noch hängiger Rechtsstreit zwischen Philip Morris (mit rechtlichem Sitz in der Schweiz) und Uruguay (als Mitglied der Rahmenkonvention der WHO) zeigt, wie ungünstig die Schweiz dastehen kann, wenn sie hier tätigen Tabakfirmen erlaubt, in der Schweiz nicht zugelassene Zigaretten in Länder zu exportieren, die diese im Einklang mit der Rahmenkonvention nicht akzeptieren. Unter Berufung auf ein Investitionsschutzabkommen zwischen der Schweiz und Uruguay von 1991 versucht Philip Morris, Uruguay zum Import solcher Zigaretten zu zwingen, und unter Umgehung uruguayischer Gerichte erhebt der Tabakkonzern nun vor einem Schiedsgericht der Weltbank Schadenersatzansprüche gegenüber Uruguay von über 2 Milliarden Dollar - beinahe 5\% des dortigen Bruttoinlandprodukts. Wie das Verfahren auch ausgeht, werde Uruguay rund 8 Millionen Dollar Gerichtskosten übernehmen müssen, wie die Alliance Sud ermittelt hat [6]. Am Rande einer Sitzung bei der WHO in Genf war letzthin zu hören, dass Philip Morris dabei durch schweizerische Anwälte beraten werde. Gewinnt Philip Morris das Verfahren gegen Uruguay, so wird in den Kreisen der Rahmenkonvention befürchtet, dass der Tabakmulti von der Schweiz aus weitere Länder mit der Drohung hoher finanzieller Verluste unter Druck setzen wird. Eine Ergänzung des TPG durch ein Produktions- und Exportverbot für in der Schweiz nicht zugelassene Zigaretten wäre einfach und könnte dies verhindern.

\section{Schlussfolgerung: Die Gesundheit der Schweizer und der Ruf der Schweiz}

«Gesundheit ist unser höchstes Gut» lautet das weltweit befolgte Sprichwort. Für die Schweiz gilt es aber zumindest im Bereich der Verhütung chronischer Krankheiten - nicht, falls die hier angeführten Probleme im Tabakproduktegesetz nicht beseitigt werden. Dazu kommt die Frage der Solidarität der Schweiz mit der internationalen Staatengemeinschaft. Wollen wir wirklich eine Schweiz, die gezielt ermöglicht, die Prävention chronischer Krankheiten in anderen Ländern zu behindern? Während im Finanzsektor ein Einlenken im Rahmen internationaler Regelungen eingeleitet wird, könnte das Tabakproduktegesetz die Möglichkeit bieten, auch im Gesundheitsbereich der internationalen Entwicklung $\mathrm{zu}$ folgen und den Ruf der fehlenden Solidarität hinter uns zu lassen. Dazu braucht es jedoch ein Gesetz, das erlaubt, das Anfixen unserer Jugend kompromisslos und wirklich wirksam zu verhindern und partnerschaftlich mit dem Rest der Welt an der Besiegung der Tabakepidemie zusammenzuarbeiten.

Für den Fall, dass die Vernehmlassung nicht zu einem solchen konsequent formulierten Gesetz führen sollte, müssten neue politische Strategien entwickelt werden, die den alten Diskurs im Sinne von «Staatliche Kontrolle vs. Freiheit» nicht mehr zulassen und der Tabakindustrie nicht mehr die Chance gibt, die Diskussion im Parlament mit Referendumsdrohungen $\mathrm{zu}$ beherrschen. Selbst das Referendum gegen ein zu schwaches Gesetz zu ergreifen, bringt die Prävention nicht weiter. Gesundheits-, Konsumenten-, Sport- und Jugendorganisationen könnten jedoch für den Fall eines ungenügenden Gesetzes mit einer gemeinsamen Volksinitiative (z. B. «Anfixerinitiative») drohen, sollte das Parlament das Ziel der Besiegung der Tabakepidemie nicht ernst nehmen. Ziel müsste eine Schweiz sein, welche die internationalen Anstrengungen und Konventionen zur Tabakkontrolle unterstützt und mitträgt und dabei gesundheitlichen gegenüber wirtschaftlichen Überlegungen den Vorrang gibt.

\section{Interaktiver Artikel}

Wollen Sie diesen Artikel kommentieren? Nutzen Sie dafür die Kommentarfunktion in der OnlineVersion oder sehen Sie nach, was Ihre Kolleginnen und Kollegen bereits geschrieben haben: www.saez.ch/aktuelle-ausgabe/interaktive-beitraege/ 